\begin{tabular}{c} 
International Journal of Engineering \& Technology, 7 (3) (2018) 1233-1235 \\
International Journal of Engineering \& Technology \\
SPC \\
Website: $\begin{array}{c}\text { www.sciencepubco.com/index.php/IJET } \\
\text { doi: } 10.14419 / \text { ijet. } 7 \text { 7i3.12486 } \\
\text { Research paper }\end{array}$ \\
\hline
\end{tabular}

\title{
Tsunami wave propagation by voronoi diagram
}

\author{
V. Yuvaraj ${ }^{1}$, S. Rajasekaran ${ }^{2}$, D. Nagarajan ${ }^{3}$ \\ ${ }^{1}$ Department of Mathematics, Sri Sairam Institute of Technology, Chennai-44 \\ ${ }^{2}$ Department of Mathematics, BS Abdhur Rahman University, Chennai48 \\ ${ }^{3}$ Department of Mathematics, Hindustan Institute of Technology \& Science, Chennai- 603103 \\ *Corresponding author E-mail: vryuva@gmail.com
}

\begin{abstract}
Cellular automata is the model applied in very complicated situations and complex problems. It involves the Introduction of voronoi diagram in tsunami wave propagation with the help of a fast-marching method to find the spread of the tsunami waves in the coastal regions. In this study we have modelled and predicted the tsunami wave propagation using the finite difference method. This analytical method gives the horizontal and vertical layers of the wave run up and enables the calculation of reaching time.
\end{abstract}

Keywords: Tsunami Wave Propagation; Voronoi Diagram; Fast Marching Method; Finite Difference Methods; Reaching Time.

\section{Introduction}

Tsunami waves are caused by the movement of the earth plates under the ocean. They create enormous disaster for people who lives near the sea shores. The prediction of tsunami waves is not accurate. Calculation of the level of impact of alert for tsunami under the earth is based on the dimensions of previously occurred earthquakes. Sometimes the Tsunami alerts fail due to wrong prediction. The study of tsunami wave simulation and modeling of wave propagation enables accurate prediction of tsunami wave occurrence. The wave propagation is based on many constraints like depth of the ocean, bathymetry, distribution of energy from the waves, wind direction, etc., Many models have been developed to predict the time that the tsunami waves reach the coastal regions, along with the calculation of the height of the waves.

Cellular automata is a mathematical model used in many complex systems and solves complex problems in a systematic and iterative manner, using cell based modeling for all the dimensional matrices. Every matrix stores the details and cells are determined by the behaviour of cells nearer to them. Each cell communicates with others without any organised administration of cells. Cellular automata is applied in many fields like robotics, physics, natural sciences, etc. The modeling of tsunami wave propagation uses cellular automata in all dimensions of the space [3]. The two-dimensional cellular automata give the propagation of the waves in the surface of the oceans. The hexagonal cellular automata model for wave establishes a detailed structure of waves under homogenous and non-homogeneous conditions [5].

\section{Analytic model}

The Voronoi diagram is the graphical method which is based on the distance between points. Plane stretch gives an option method for making calculation for the voronoi diagram of $\mathrm{n}$ points in the plane for bounded time and direct space. The entire space is partitioned into groups of voronoi areas spatial object by the voronoi model. This Model shows the expansion of the regions around the objects into a common region connected to the unique point for every partitioned area. In a well-defined Space S, distance is considered as function $T$. Let $I$ be the collection of basis for the region $i \in I$. $A_{i}$ be the subset in the defined space $S$. The voronoi partition $T_{i}$ related to the $A_{i}$ is the collection of points whose spread is not more than spread of other section $A_{j}$, where the $j$ is different index from ' $i$ '.

$\mathrm{T}_{\mathrm{i}}=\left\{\mathrm{k} \in \mathrm{S} \mid \mathrm{d}\left(\mathrm{k}, \mathrm{A}_{\mathrm{j}}\right) \geq \mathrm{d}\left(\mathrm{k}, \mathrm{A}_{\mathrm{i}}\right)\right.$ forall different $\left.\mathrm{i} \in \mathrm{I}\right\}$

Weighted Voronoi Diagram in Wave Propagation

The space is a finite dimensional space which contains many infinite points (spread). The points over the region are unique in the voronoi cells. The region is considered as a convex plane polygon. The wave spread region in the ocean is considered as the finite dimensional space. the weighted voronoi cell is executed for the three-dimensional space [17]. Weight is assigned for the depth of the water in the tsunami wave. The crystal voronoi diagram is implemented for the increase of wave height the growth based on the speed of the wave. The weight (the depth of the water level) for every spread (point) $M_{i}$ is defined as

$T_{i}=\left\{k \in S \mid d\left(k, A_{j}\right) / M_{j} \geq d\left(k, A_{i}\right) / M_{i}\right.$ forall different $\left.i \in I\right\}$

Tsunami waves occur from various sources they interact and produce a new wave propagation. This wave propagation is patterned in voronoi diagram [8]. At the time ' $t$ ', the pattern can be identified, and the boundary is fixed with a common point. The speed of the wave propagation is fixed as constant for some $\Delta t$. Every wave from the source wave is identified with a distance $\mathrm{T}_{\mathrm{i}}$ for the time $\mathrm{t}$. From the weighted voronoi diagram, equidistant pair of waves has been found with respect to the source wave. 


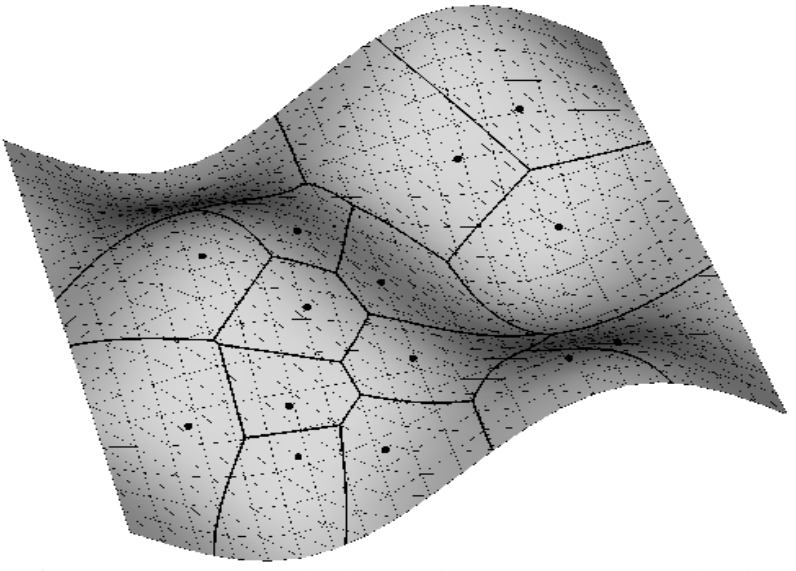

Fig. 1: The Sample Voronoi Diagram for the Wave Propagation in the Ocean.

Wave propagation in the sea region is partitioned as voronoi cells based on Cellular automata methodology [7]. Propagation for each wave is associated with the relevant region featured by the distance from the common point.

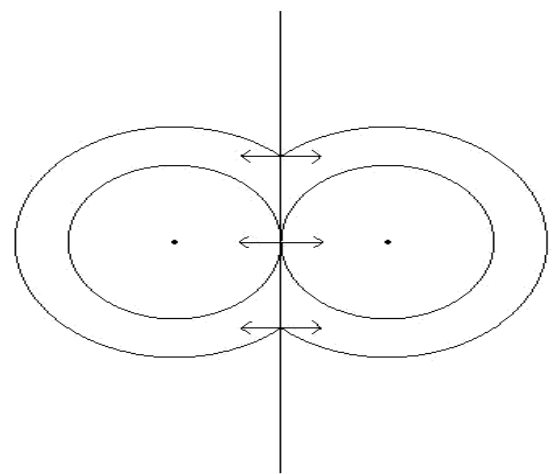

Fig. 2: The Common Axis between the Two-Wave Spread by Voronoi Diagram.

Every region in the voronoi diagram for wave spread is linked with the Cellular automata as for individual cells. Each cell has wind, speed of wave, the density and depth.

For any point $\mathrm{K}$ in the wave spread in co-ordinate plane $\left(\mathrm{x}^{\prime}, \mathrm{y}^{\prime}\right)$ then we get an equation. Let $w=\frac{M_{1}}{M_{2}}$. given that $M_{1}>M_{2}$, where $M_{1}$ and $M_{2}$ are two weights at the spread $A_{1}$ and $A_{2}$.

$\left(\mathrm{x}^{\prime}-\frac{\mathrm{x}}{\left(\mathrm{w}^{2}-1\right)}\right)^{2}+\mathrm{y}^{\prime 2}-\frac{\mathrm{w}^{2}}{\left(\mathrm{w}^{2}-1\right)^{2}} \mathrm{x}^{2}=0$

The above equation represents the circle generated by the points $\mathrm{A}_{1}$ and $\mathrm{A}_{2}$ with constant ratio w.

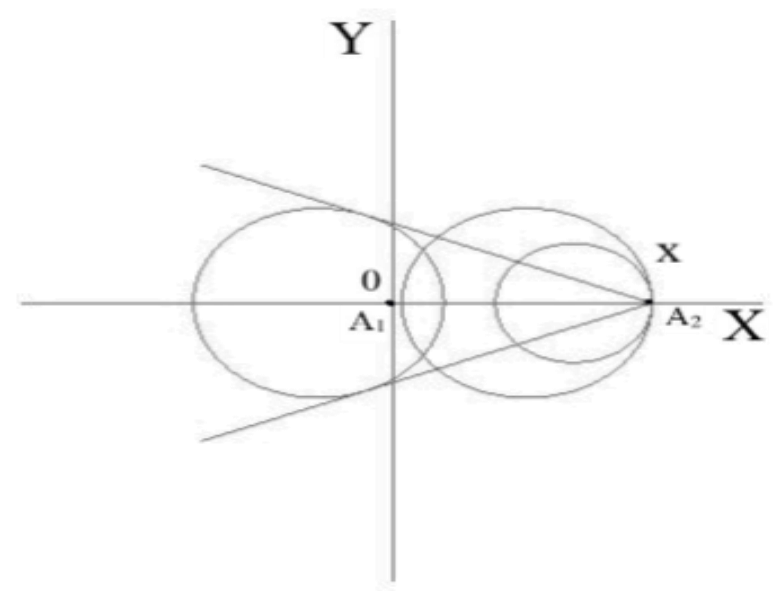

Fig. 3: The Two-Point Wave Spread by Voronoi Diagram.

\section{Fast marching method}

Fast marching method approaches the tsunami wave propagation in the ocean with stationary and steady conditions. The region is considered as the grid point. For every point the wave crosses the time $\mathrm{t}$ has been recorded for every grid points $\mathrm{T}_{\mathrm{s}}=$ $\left\{t_{1}(x, y), t_{2}(x, y), t_{3}(x, y), ..\right\}$ w.r.to the coordinate points $(x, y) . T_{s}$ is time function which represents the time of wave crossing the points $(\mathrm{x}, \mathrm{y})$ for every primary grid regions. The time function $\mathrm{T}_{\mathrm{s}}$ looks like an irregular cone for every wave propagation surface. Time function identifies the wave front positions every time. The consolidated time function gives the level set considered as height.

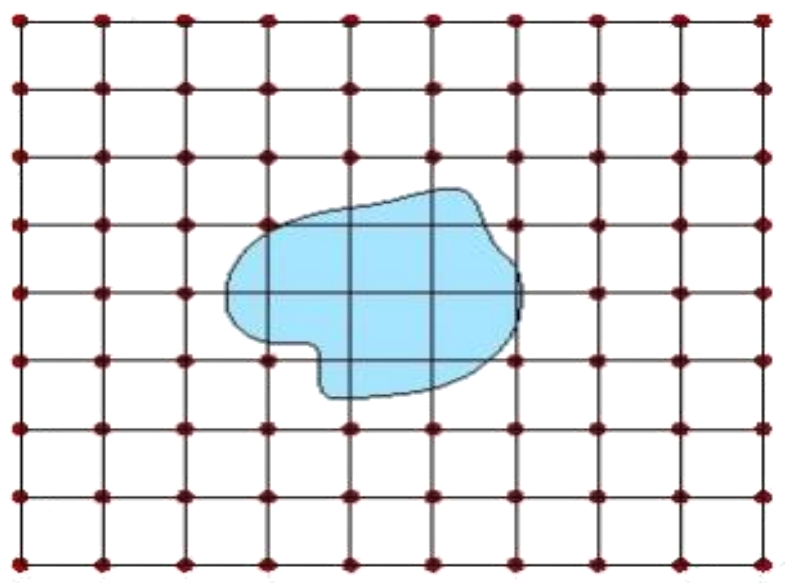

Fig. 4: The Wave Propagation over the Grid Regions.

The function is constructed for various levels of the wave propagation surface. The steady wave propagation speed can be calculated with respect to the front moving time point function. Consider the non-linear differential equation for the wave propagation model.

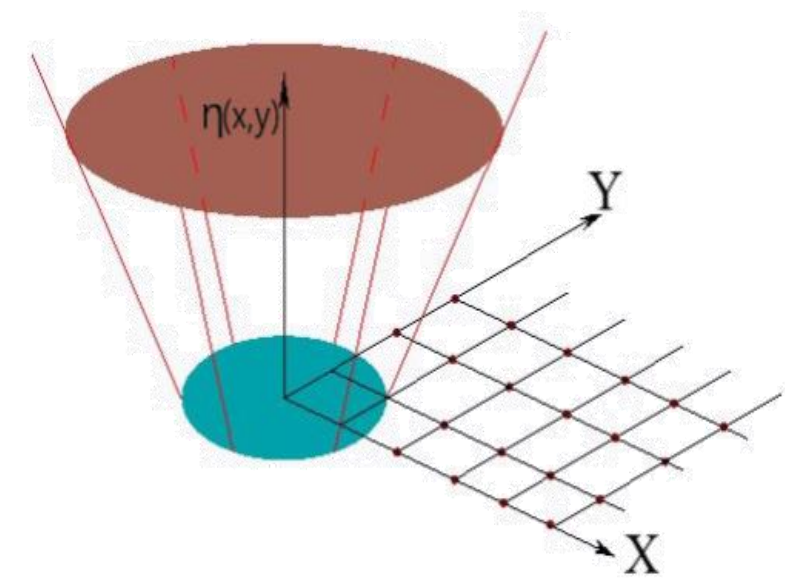

Fig. 5: Wave Front Moving Time Point Function in (X, Y) Plane.

Let $\Lambda$ be the region with boundaries in the defined plane $\mathrm{R}^{3}$. The function which has real values is considered as $\eta(x, y)>0$ for any $(\mathrm{x}, \mathrm{y})$ in $\Lambda$. Another function $\delta(\mathrm{x}, \mathrm{y})$ is also defined under the region $\Omega$.

The non-linear differential equation is $\left|\nabla^{2} \alpha(\mathrm{x}, \mathrm{y})\right|=\eta(\mathrm{x}, \mathrm{y})$ with the condition $\alpha(\mathrm{x}, \mathrm{y})=\delta(\mathrm{x}, \mathrm{y})$ in the region $\Omega$. The values of $\eta(x, y)$ and $\delta(x, y)$ are defined to find the $\alpha(x, y)$. The inverse of $\eta(x, y)$ represent the velocity of the tsunami wave propagation and initially $\delta(\mathrm{x}, \mathrm{y})$ as zero.

The solution of $\alpha(\mathrm{x}, \mathrm{y})$ represents the time taken to reach the region nearer for every point $(\mathrm{x}, \mathrm{y})$ in $\Lambda$. Using the finite difference method, the time function can be found for every region by the collection of values at the different points $\alpha_{\mathrm{t}, \mathrm{i}, \mathrm{j}}=\alpha(\mathrm{dt}, \mathrm{idx}, \mathrm{jdy})$ 
idx, jdy and dt are the increment of the (x, y) and time t. Other technique is applied to find $\alpha_{t, i, j}$ by step by step method with analysing the points nearer to the region.

\section{Numerical approximation}

Consider the grid values of the region $\Lambda$.

$\sup \left(\mathrm{T}_{\mathrm{t}, \mathrm{i}, \mathrm{j}}^{-\mathrm{x}} \alpha_{\mathrm{t}, \mathrm{i}, \mathrm{j}}-\mathrm{T}_{\mathrm{t}, \mathrm{i}, \mathrm{j}}^{+\mathrm{x}} \alpha_{\mathrm{t}, \mathrm{i}, \mathrm{j}}, 0\right)^{2}+\sup \left(\mathrm{T}_{\mathrm{t}, \mathrm{i}, \mathrm{j}}^{-\mathrm{y}} \alpha_{\mathrm{t}, \mathrm{i}, \mathrm{j}}-\mathrm{T}_{\mathrm{t}, \mathrm{i}, \mathrm{j}}^{+\mathrm{y}} \alpha_{\mathrm{t}, \mathrm{i}, \mathrm{j}}, 0\right)^{2}=$ $\eta_{t, i, j}$

$\alpha_{\mathrm{x}}\left(\mathrm{x}_{\mathrm{tij}}\right)=\mathrm{T}_{\mathrm{t}, \mathrm{i}, \mathrm{j}}^{-\mathrm{x}} \alpha_{\mathrm{t}, \mathrm{i}, \mathrm{j}}=-\frac{\alpha_{\mathrm{t}+1, \mathrm{i}+1, \mathrm{j}}-\alpha_{\mathrm{t}, \mathrm{i}, \mathrm{j}}}{\lambda}$

$\alpha_{\mathrm{x}}\left(\mathrm{x}_{\mathrm{tij}}\right)=\mathrm{T}_{\mathrm{t}, \mathrm{i}, \mathrm{j}}^{+\mathrm{x}} \alpha_{\mathrm{t}, \mathrm{i}, \mathrm{j}}=-\frac{\alpha_{\mathrm{t}+1, \mathrm{i}+1, \mathrm{j}}-\alpha_{\mathrm{t}, \mathrm{i}, \mathrm{j}}}{\lambda}$

$\alpha_{\mathrm{y}}\left(\mathrm{x}_{\mathrm{tij}}\right)=\mathrm{T}_{\mathrm{t}, \mathrm{i}, \mathrm{j}}^{-\mathrm{y}} \alpha_{\mathrm{t}, \mathrm{i}, \mathrm{j}}=-\frac{\alpha_{\mathrm{t}+1, \mathrm{i}, \mathrm{j}+1}-\alpha_{\mathrm{t}, \mathrm{i}, \mathrm{j}}}{\lambda}$

$\alpha_{\mathrm{y}}\left(\mathrm{x}_{\mathrm{tij}}\right)=\mathrm{T}_{\mathrm{t}, \mathrm{i}, \mathrm{j}}^{+\mathrm{y}} \alpha_{\mathrm{t}, \mathrm{i}, \mathrm{j}}=-\frac{\alpha_{\mathrm{t}+1, \mathrm{i}, \mathrm{j}+1}-\alpha_{\mathrm{t}, \mathrm{i}, \mathrm{j}}}{\lambda}$

Its shown that when $\alpha_{N}=\inf \left(\alpha_{t, i-1, j}, \alpha_{t, i+1, j}\right) \quad, \quad \alpha_{M}=$ $\inf \left(\alpha_{t, i, j-1}, \alpha_{t, i, j+1}\right)$ and $\left|\alpha_{N}-\alpha_{M}\right| \leq \lambda \eta_{t, i, j}$

$\alpha_{t, i, j}=\frac{\alpha_{N}+\alpha_{M}}{2}+\frac{1}{2}\left(\left(\alpha_{N}+\alpha_{M}\right)^{2}-2\left(\alpha_{N}^{2}+\alpha_{M}^{2}-\left(\lambda \eta_{t, i, j}\right)^{2}\right)\right)^{1 / 2}$

The time wave function can be calculated, and the results are shown for different regions. Wave propagation generation is identified from the results and reaching time for the waves can be calculated efficiently.

\section{Conclusion}

This paper presents the tsunami wave propagation in ocean and seashore as identified by the application of applying the concept of voronoi diagram and cellular automata. First, the partition of the wave propagation area in the grid region is shown using weight voronoi diagram. Then the wave spread time function is calculated using the fast march method and finite difference method. This method enables the efficient and exact time valued function at the time of tsunami wave on all the region of the ocean. It minimizes the complexity of computation in the wave propagation and provides an efficient analytic model for the tsunami wave propagation.

\section{References}

[1] F. Diasa, D. Dutykhc, L. O’Briena, E. Renzia, T. Stefanakisb, On the modelling of tsunami generation and tsunami inundation, Procedia $\begin{array}{llllll}\text { IUTAM } & 10 & (2014) & 338 & - & 355\end{array}$ https://doi.org/10.1016/j.piutam.2014.01.029.

[2] B Gutenberg, Tsunamis and earthquakes, Bulletin of the Seismological Society of America 29 (1939) 517-526.

[3] D Dutykh, D Mitsotakis, L B Chubarov, On the contribution of the horizontal sea-bed displacements into the tsunami generation process, Ocean Modeling $56 \quad$ (2012) 43-56. https://doi.org/10.1016/j.ocemod.2012.07.002.

[4] A Ezerky, N Abcha, E Pelinovsky, Physical simulation of resonan wave run-up on a beach, Nonlinear Processes Geophysics 20 (2013) 35-40. https://doi.org/10.5194/npg-20-35-2013.

[5] P Brufau, M E Va'zquez-Ce'ndon, P Garci'a-Navarro, A numerical model for the flooding and drying of irregular domains, International Journal for Numerical Methods in Fluids 39 (2003) 247-275. https://doi.org/10.1002/fld.285.

[6] D L George, R J LeVeque, Finite volume methods and adaptive refinement for global tsunami propagation and local inundation, Sci. Tsunami Hazard 245 (2006) 319-328.

[7] A I Adamatzky, Voronoi-Like Partition of Lattice in Cellular Automata, Mathematical Computation Modeling 234 (1996) 51-66. https://doi.org/10.1016/0895-7177(96)00003-9.
[8] S Stifter, An axiomatic approach to Voronoi-diagrams in 3D, Journal of Computer and system science 43 (1991) 361-379. https://doi.org/10.1016/0022-0000(91)90019-2.

[9] J Chen, Voronoi special dynamic model, Mapping press, (2002) 2437.

[10] Yongze Chen, Philip L.-F. Liu, Modified Boussinesq Equations and Associated Parabolic Model for Water Wave Propagation, Journal of Fluid Mechanics $228 \quad$ (1995) 351-381. https://doi.org/10.1017/S0022112095001170.

[11] W Kristina, O Bokhove, E Van Groesen, Effective coastal boundary conditions for tsunami wave run-up over sloping bathymetry, Nonlinear Processes Geophysics 21 (2014) 987-1005. https://doi.org/10.5194/npg-21-987-2014.

[12] G Klopman, E Van Groesen, M A Dingemans, Variational approach to Boussinesq modelling of fully non-linear water waves, J. Fluid Mechanics $657 \quad$ (2010) 36-63 https://doi.org/10.1017/S0022112010001345.

[13] Kristina, W., Van Groesen, E., and Bokhove, O, Effective Coastal Boundary Conditions for Dispersive Tsunami Propagation, Memorandum 1983, Department of Applied Mathematics, University of Twente, Enschede, the Netherlands (2012).

[14] S Aggelos, Dimakopoulos, Antonella Guercio, Giovanni Cuomo, Advanced numerical modelling of tsunami wave propagation, transformation and run-up, Proceedings of the Institution of Civil Engineers Engineering and Computational Mechanics 167 (2014) 3.

[15] Phung DangHieua, Tanimoto Katsutoshia, Vu Thanh Ca, Numerical simulation of breaking waves using a two-phase flow model, Applied

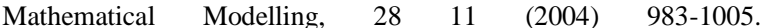
https://doi.org/10.1016/j.apm.2004.03.003.

[16] Tetsushi Nishida, Kokichi Sugihara, Masato Kimura, Stable markerparticle method for the Voronoi diagram in a flow field, Journal of Computational and Applied Mathematics 202 (2007) 377 - 391 https://doi.org/10.1016/j.cam.2006.01.035.

[17] F Aurenhammer, H Edelsbrunner, An optimal algorithm for constructing the weighted Voronoi diagram in the plane, Pattern Recognition 17 (1984) 251-257. https://doi.org/10.1016/00313203(84)90064-5.

[18] Cecilia Bohler, Rolf Klein, Andrzej Lingas, Chih-Hung Liu, Forestlike abstract Voronoi diagram in linear time, Computational Geometry: Theory and Applications 68 (2018) 134-145. https://doi.org/10.1016/j.comgeo.2017.06.013.

[19] Santiago Garrido, Luis Moreno, Fernando Martin, and David Alvarez, Fast Marching subjected to a Vector Field - path planning method for Mars rovers, Experts system and its applications $78 \mathrm{C}$ (2017) 334-346.

[20] F. Harlow, J Welch, Numerical calculation of time-dependent viscous incompressible flow of fluid with free surface, Physics Fluids 8 (1965). 2182-2189. https://doi.org/10.1063/1.1761178.

[21] Li Jin, Donguk Kim, Lisen Mu, Deok-Soo Kim, Shi-Min Hu, A sweepline algorithm for Euclidean Voronoi diagrams of circles, Elsevier Computer-Aided Design 38 (2006) 260-272. https://doi.org/10.1016/j.cad.2005.11.001.

[22] Tetsushi Nishida, Kokichi Sugihara, Voronoi diagram in the flow, Algorithms and Computation, 14th International Symposium, ISAAC 2003, Kyoto, December 2003, Lecture Notes in Computer Science, Springer 2906 (2003) 26-35.

[23] Sugihara K, Voronoi diagrams in a river, International Journal of Computational Geometry and Application 2 (1992) 29-48. https://doi.org/10.1142/S0218195992000032. 